\title{
Alterations of Tooth Eruption and Growth in Pups Suckling from Diabetic Dams
}

\author{
MARIANO E. VILLARINO, JUAN A. GOYA, ROMINA C. DE LUCCA, AND ANGELA M. UBIOS \\ Department of Histology and Embryology. School of Dentistry. University of Buenos Aires, \\ Buenos Aires (C1122AAH), Argentina
}

\begin{abstract}
ABST
Several studies have confirmed a decrease in the quality and
quantity of milk of mothers with diabetes during lactation.
However, it remains unclear how maternal diabetes affects the
offspring specifically during lactation. The aim of this study was
to evaluate body and mandibular growth and tooth eruption in
pups suckling from diabetic dams. The study was performed on
13 Wistar rat pups that were born to dams that were subjected to
experimental diabetes on the day of parturition. Body weight and
body size were recorded regularly throughout the study. The
experimental pups and a group of eight age-matched pups suck-
ling from nondiabetic dams were killed at weaning. Both hemi-
mandibles were excised and fixed. Right hemimandibles were
radiographed to assess mandibular growth and tooth eruption.
The left hemimandibles were processed to obtain buccolingually
oriented sections at the level of the first mesial root of the first
\end{abstract}
Diabetes encompasses a group of metabolic diseases characterized by hyperglycemia, which results from decreased insulin secretion, from functional deficiency of insulin action, or both. Diabetes-associated chronic hyperglycemia causes dysfunction, long-term damage, and failure of several body organs (1).

When a mother has diabetes, the alterations in maternal metabolism cause damage to the offspring, not only during gestation but also during lactation. Alterations in maternal metabolism during gestation are responsible for fetal macrosomia and congenital malformations, among other consequences $(2,3)$. There are reports of the long-term effects of neonatal breastfeeding in children of mothers with pregestational diabetes during pregnancy (4). It may result in an increased risk for becoming overweight and developing impaired glucose tolerance during childhood. Moreover, diabetic dams have decreased yield and altered composition of the milk

Received August 10, 2004; accepted February 11, 2005.

Correspondence: Angela Matilde Ubios, D.D.S., Ph.D., Department of Histology and Embryology. School of Dentistry, University of Buenos Aires, Marcelo T. de Alvear 2142 1st A., Buenos Aires (C1122AAH), Argentina; e-mail: amubios@histo.odon.uba.ar.

This work was supported in part by grant UBACyT O013 from the University of Buenos Aires.

DOI: 10.1203/01.PDR.0000180599.54807.24 lower molar. Histologic and histomorphometric studies were performed. Results showed that body weight and body size were significantly lower in experimental animals at weaning compared with their age-matched controls. Smaller mandible size and reduced tooth eruption in experimental animals compared with controls were observed. The length, width, and bone volume of the developing alveolus were reduced in experimental animals compared with controls. The results obtained in this study allow the conclusion that suckling from diabetic dams results in reduced body, mandible size, and tooth eruption of the pups at weaning. (Pediatr Res 58: 695-699, 2005)
Abbreviation
EGF, epidermal growth factor

(5-9). Alterations in maternal metabolism caused by diabetes during lactation may affect the offspring specifically when the known effects that maternal diabetes has on their pups during pregnancy are excluded. Thus, the first aim of this study was to measure the effect of reduced milk quality and quantity on body size and weight of the pups of dams that were rendered diabetic before lactation.

Tooth eruption is a complex process in developmental biology and is yet to be fully understood. Tooth and periodontal development are two major events associated to this process and in which the dental follicle plays a key role: the root is shaped involving dentin and cementum formation, the bundle bone of the developing alveolus is formed, and collagen fibers are inserted in bone and cementum, forming the periodontal ligament. It is evident that bone formation and bone resorption play an important role during this period (10-12). As tooth eruption takes place, the maxillaries and the jaw bones continue to grow $(13,14)$. It has been demonstrated that bone formation is involved in tooth eruption and that the alterations in bone formation affect tooth eruption and mandibular growth (15-19). The second aim of the study was to determine whether jaw bone growth and tooth eruption were affected in pups by the alterations in milk quality and quantity of diabetic mothers during lactation. 


\section{METHODS}

Thirteen newborn Wistar rat pups were used. Their dams were subjected to experimental induction of diabetes on the day of parturition by administration of a single i.p. dose of $60 \mathrm{mg} / \mathrm{kg}$ body weight of streptozotocin (SIGMAALDRICH, Inc., St. Louis, MO) dissolved in freshly prepared citrate buffer (pH 4.0) (20). Hyperglycemia and glycosuria were determined $24 \mathrm{~h}$ after injection. Glycemia was determined following the glucose-oxidase method using a blood glucose meter (Prestige LX; HDI-Home Diagnostics, Inc., Ft. Lauderdale, FL). Glycosuria was monitored using a colorimetric method by means of reagent strips (Diastix; Bayer Argentina, Buenos Aires, Argentina). Animals that presented glycemia levels $>300 \mathrm{mg} / \mathrm{dL}$ and glycosuria (500$1000 \mathrm{mg} / \mathrm{dL}$ ) were considered diabetic. Eight age-matched pups of dams that received an injection of an equivalent dose of citrate buffer on the day of parturition served as controls. The pups were killed by ethyl ether overdose at weaning ( $21 \mathrm{~d}$ of age). The National Institutes of Health Guidelines for the Care and Use of Laboratory Animals (National Institutes of Health publication 85-23 Rev. 1985) were observed.

Body weight and size (head-to-tail length) of control and experimental animals were recorded daily throughout the study. From each rat, the left and right hemimandibles were excised and fixed in $10 \%$ formalin. Right hemimandibles were radiographed to assess mandibular growth (21) (Fig. 1A). Measurements were performed on projections of the lateral jaw radiographs. Alteration of growth of the vertical and horizontal rami of the mandible were determined by measuring the gonial angle (15). Tooth eruption was also measured using projections of these radiographs (16). Figure $1 B$ illustrates that eruption of the first and second molars was assessed as the distances from the

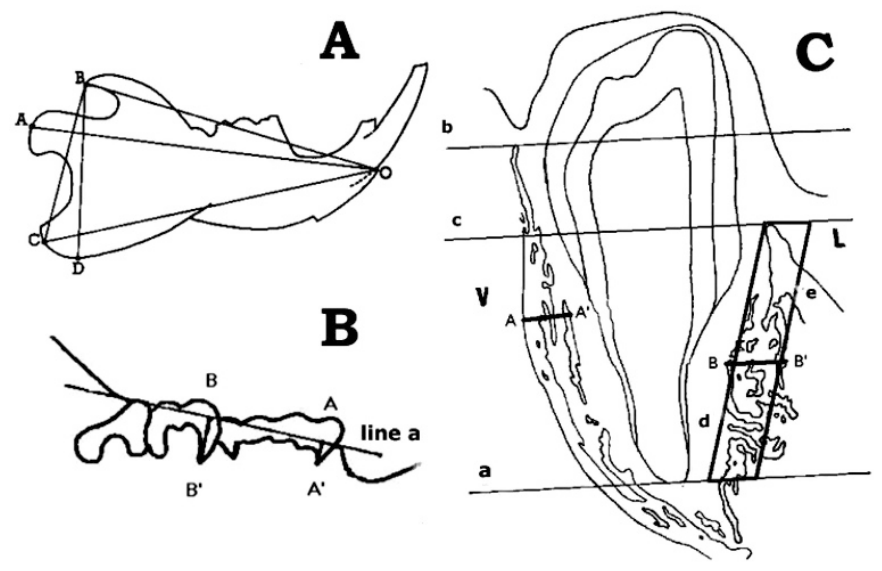

Figure 1. Method for the histologic, histomorphometric, and morphometric studies. (A) Mandibular length was measured from the most anterior inferior bone point to the middle of the condyle (length O-A). The base of the jaw was considered from the most anterior inferior bone point $(\mathrm{O})$ to the most posterior point of the gonion $(\mathrm{C})$, and the height of the jaw was considered from the most superior posterior point of the coronoid process (B) to the inferior border of the inferior ramus (D). We also measured mandibular area, which was calculated from a triangle formed among three stable points: the most superior posterior point of the coronoid process (B), the most posterior point of the mandible (C), and the most anterior inferior bone point $(\mathrm{O})$. $(B)$ Evaluation of tooth eruption. Line a is drawn from the mesial crest of the first molar alveolus to the intersection of the anterior border of the mandibular ramus and the third developing molar; segments are traced perpendicular to line a to reach the mesial cementoenamel junction of the first (segment $\mathrm{AA}^{\prime}$ ) and second molars (segment BB'). (C) Method used to perform the histomorphometric study of the developing alveolus. Segment A-A', parallel to and equidistant from line a and $b$, drawn through the vestibular plate; segment B-B', parallel to and equidistant from line a and c, drawn through the lingual plate; line a, straight line joining the tips of Hertwig's epithelial root sheath; line b, line parallel to line a and drawn through the upper point of the vestibular bone crest; line c, parallel to $a$ and $b$ and drawn through the upper point of the lingual bone crest; line $\mathrm{d}$, straight line joining the upper point of the lingual bone crest and point $\mathrm{B}$; line e, line drawn $1 \mathrm{~cm}$ to the right of and parallel to line d; segment A-A', parallel to and equidistant from line $\mathrm{a}$ and $\mathrm{b}$, drawn through the vestibular plate; segment B-B', parallel to and equidistant from line a and c, drawn through the lingual plate. mesial cementoenamel junctions to a baseline drawn between the landmarks of the crest of the alveolus on the mesial aspect of the first molar to the point where the anterior border of the vertical ramus intersected the third molar tooth germ (16). The distances $\mathrm{AA}^{\prime}$ and $\mathrm{BB}^{\prime}$ were measured in $\mathrm{mm}$ at $\times 12$ magnification on the projected radiographs. The measurements then were reduced to real scale. All tooth eruption measurements were negative values, and greater distances suggested less eruption.

The left hemimandibles were decalcified in EDTA and processed using routine histologic techniques to obtain coronal sections at the level of the first mesial root of the lower first molar. Histologic and histomorphometric studies of the developing alveolus (17) were performed (Fig. 1C). The following histomorphometric parameters were assessed on the developing dental alveolus according to stereologic principles (22):

Length of vestibular plate: distance between lines $a$ and $b$.

Width of vestibular plate: distance between points $\mathrm{A}$ and $\mathrm{A}^{\prime}$.

Bone volume of vestibular plate: fraction of the total volume corresponding to bone tissue. Total volume was taken as bone tissue plus bone marrow in the vestibular plate.

Length of lingual plate: distance between lines a and c.

Width of lingual plate: distance between points B and B'.

Bone volume of the lingual plate: fraction of the total volume corresponding to bone tissue, considered in the area between lines a, c, d, and e. Total volume was taken as bone tissue plus bone marrow in the lingual plate.

Bone activity in bundle bone of the vestibular plate of the developing alveolus was also studied, considering

Sob/BS (\%): bone formation: fraction of total bone surface with active osteoblasts.

ES/BS (\%): bone resorption: erosive surface as a fraction of total bone surface.

LCS (\%): lining cells surface: fraction of total bone surface with inactive osteoblasts.

Statistical analysis. One-way ANOVA was used to assess the statistical significance of the results. All results were expressed as the mean \pm SEM

\section{RESULTS}

At parturition, body weights and sizes of the pups were similar in both groups. Pups suckling from diabetic rats had significantly lower body weights at weaning compared with their age-matched controls. This difference between groups was highly significant at day 7 and was consistently more pronounced at $21 \mathrm{~d}$ (Fig. 2). Body size at weaning was significantly lower in experimental animals compared with their age-matched controls. This difference reached high statistical significance at day 7 (Fig. 2).

Evaluation of mandibular growth showed a statistically significant diminution in length, base, height, and area $(p \leq$ 0.001) of experimental animals at weaning compared with controls. However, no differences were found between groups when the gonial angle was considered (Table 1).

In the experimental group, tooth eruption was delayed at weaning compared with controls. The differences observed in tooth eruption were statistically significant on both the first (segment $\mathrm{AA}^{\prime} ; p \leq 0.001$ ) and second molars (segment $\mathrm{BB}^{\prime} ; p$ $\leq$ 0.01; Table 1). Accordingly, the histologic study showed that the tooth crown of 21-d-old control animals had emerged. Although the gingival crest had not acquired its definite shape, the vestibular bone crest was level with the cervical third of the crown. Root formation of the apical third was complete, but the apical foramen was open widely. In contrast, a thin layer of mucosa and reduced dental epithelium covered the developing crown in the experimental group. The vestibular bone crest still covered the middle third of the crown. Minor root development was observed compared with the controls. In the alveolar bone of experimental animals, the marrow cavities contained fatty marrow compared with the hemopoietic marrow found in the controls (Fig. 3). 

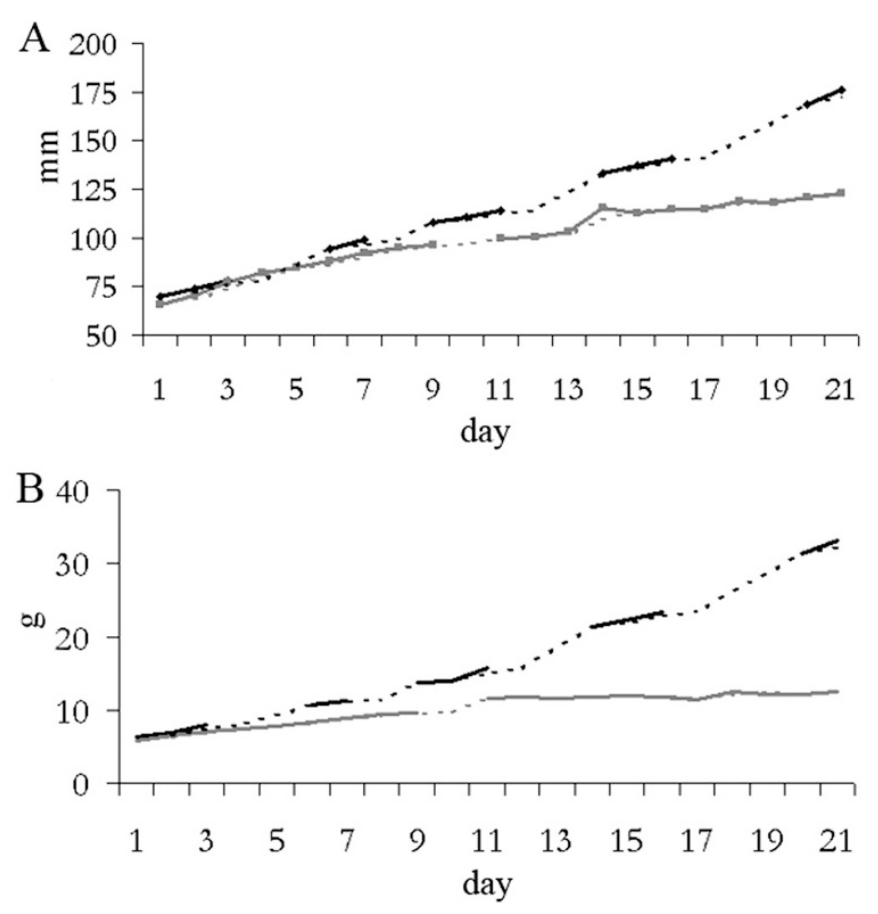

Figure 2. Body weight $(A)$ and body size $(B)$ were significantly lower in experimental animals (gray) at weaning compared with controls (black) $(n=21)$.

Length and width of the vestibular plate of the developing dental alveolus were found to be significantly different between groups (Fig. 4). Both parameters were significantly lower in experimental animals at weaning compared with the controls. Bone volume of the vestibular plates of experimental animals at weaning were also significantly lower than controls. Highly significant differences were also found between groups, as regards length and width of the lingual plate of the developing dental alveolus at weaning (Fig. 4), with pups of diabetic dams exhibiting lower values than controls. Bone volumes of the lingual plate were also significantly lower in experimental animals compared with controls.

Less bone formation and greater bone resorption were observed in experimental animals at weaning than in their agematched controls. These differences did not reach statistical significance (bone formation: control $41.98 \pm 10.63 \%$; experimental $31.33 \pm 4.72 \%$; and bone resorption: control $32.21 \pm$ $8.69 \%$; experimental $34.67 \pm 4.62 \%$ ).

\section{DISCUSSION}

In our study, the overall diminution in mandibular growth observed in pups suckling from diabetic dams correlated with the diminution in body weight and body size. However, no differences were found between groups as regards the gonial angles, indicating that the direction of growth in experimental animals was not altered by treatment. As a number of authors have reported decreased yield and/or quality of the milk of diabetic dams, it therefore is suggested that the lack of overall growth was caused by pup malnutrition (5-9). Studies performed by others, in animals that were subjected to varying degrees of protein-energy malnutrition early in life, have reported growth diminution in keeping with our results (23). Together, these data suggest that overall diminution in body and mandibular growth observed in this study could be the consequence of malnutrition.

The experimental conditions used in this study produced results that are due exclusively to the effect of breastfeeding from dams that were rendered diabetic after parturition. Both alveolar bone growth and tooth eruption are markedly decreased in pups suckling from diabetic dams.

Thulesen et al. (20) observed no differences in the time of eruption of the incisor, which has continuous growth, when the pups of diabetic dams that remained untreated during lactation, treated diabetic dams, and control dams were compared. In the present study, tooth eruption was measured in rat molars teeth with limited tooth eruption, as is the case of humans. Pups suckling from diabetic dams were found to have decreased tooth eruption. Tooth eruption has similarly been decreased in other studies on the effect of intoxication with iron (17) and uranium (18) and in systemic bone conditions (19). In both of these studies and in ours, the decrease in tooth eruption was associated with delayed tooth development and less bone formation. The findings may relate to insulin deficiency in lactating rats, which causes a decrease in lactational performance, causing malnutrition (20).

High concentrations of growth factors are present in milk of various species, including human. Two of these are epidermal growth factor (EGF) and IGF-1 and are thought to play a role in the modulation of cellular proliferation and differentiation during tooth morphogenesis and eruption. The responsiveness to EGF in tooth development was proved to be stage dependent in vitro (24) and in vivo (25), and it has been shown to accelerate the time of incisor tooth eruption when exogenously administered to newborn rats (26). It has been reported that both growth hormone and IGF-1 play a main role in odontogenesis in vitro (27) and on the differentiation and growth of cartilage, bone, and hard tissues of the tooth; it has been suggested that bone morphogenetic proteins may mediate the local osteogenic actions of growth hormone and IGF-1 (28).

Table 1. Morphometric measurements of mandibular growth and tooth eruption

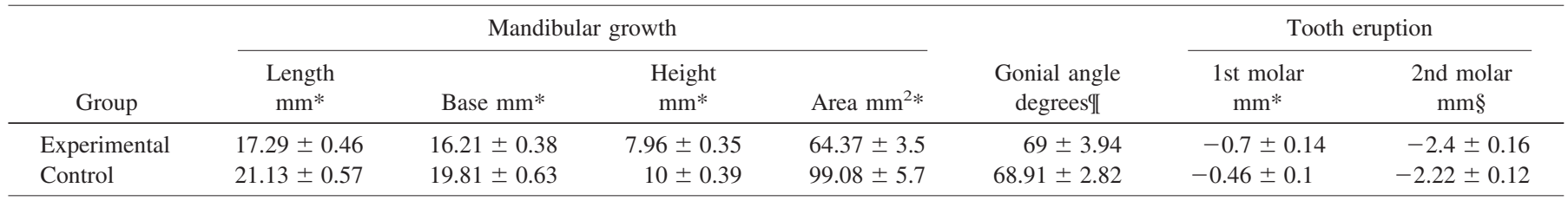

$* p \leq 0.001$

$\S p \leq 0.01$

If no statistical significance

$n=21$ 


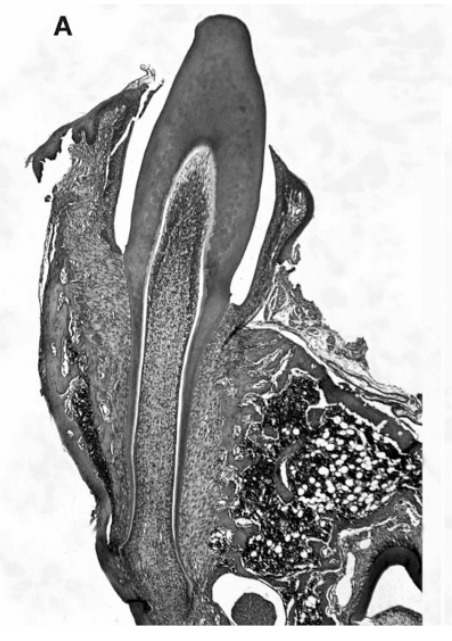

B

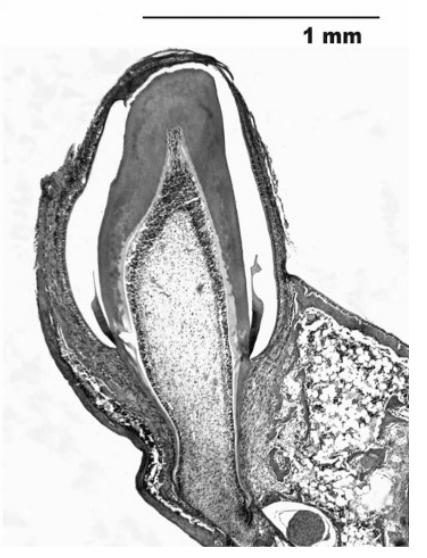

Figure 3. Histologic evaluation showed that tooth crown of 21-d-old control animals had erupted. Experimental histologic sections exhibited a thin layer of buccal mucosa and reduced dental epithelium covering the developing crown.
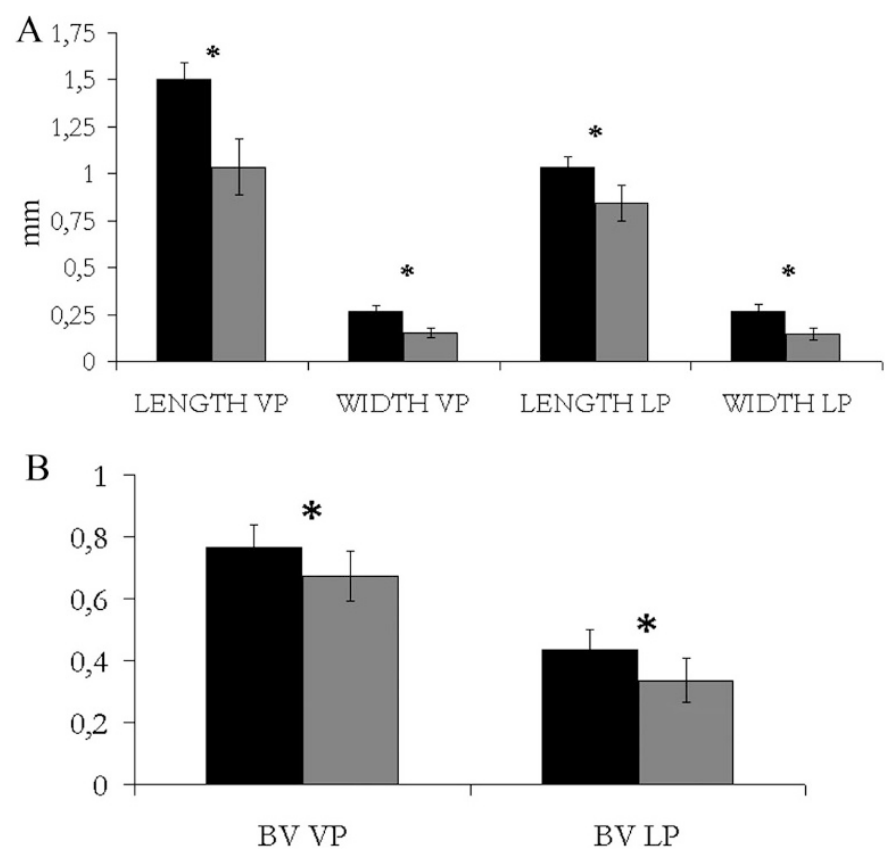

Figure 4. Length and width $(A)$ and bone volume $(B)$ of the vestibular and lingual plate were significantly lower in experimental animals (圈) at weaning compared with controls $(\boldsymbol{\square})$. LENGTH VP, length of the vestibular plate; WIDTH VP, width of the vestibular plate; LENGTH LP, length of the lingual plate; WIDTH LP, width of the lingual plate; BV VP, bone volume in the vestibular plate; BV LP, bone volume in the lingual plate $\left({ }^{*} p \leq 0.01 ; n=21\right)$.

The concentration of EGF in milk of diabetic rats is significantly decreased, as has been reported in the literature (20). To our knowledge, no studies have reported an alteration in the concentration of IGF-1 in milk of diabetic dams; however, Baumrucker et al. (29) reported that the diminution in the concentration of IGF-1 in milk correlated with a diminution in blood IGF-1 concentration in calves (29). Furthermore, it is well documented that blood IGF-1 concentration is diminished in patients with diabetes. According to the aforementioned data, a correlation between the alterations in IGF-1 and EGF in the milk of diabetic dams and the diminution in both mandibular and body growth and in tooth eruption observed in the pups should not be ruled out.

The delays in tooth formation and in eruption were paralleled by lack of development of the periodontium and the alveolar bone in the experimental group. The histomorphometric determinations of length, width, and bone volume of the vestibular and lingual plate showed that experimental animals had significantly lower values than controls. Therefore, it is reasonable to infer that bone development must have been significantly lower in experimental animals during the experimental period. Bone activity was studied using static histomorphometry, so the results provide information only about bone formation at the time of determination, i.e. at the end of the experimental period. At this time point, the difference observed between experimental and control animals as regards bone activity was not statistically significant.

As considerable alterations in colostrum and transitional milk are found in human mothers who have diabetes with good metabolic control $(4,8)$, the indication of a supplement diet during lactation should be considered to prevent the adverse effects reported here. However, there is strong evidence in the literature that breastfeeding decreases the incidence and/or severity of diarrhea, lower respiratory infection, otitis media, bacteriemia, bacterial meningitis, botulism, etc. in children (30), and that is why it is highly encouraged, even in women with both preexisting diabetes and gestational diabetes. It has been reported that after delivery, glycemic control in diabetic women deteriorates (31). Considering the protective effect of breastfeeding, strict metabolic control of mothers with diabetes during breastfeeding as well as during gestation would seem recommended, to prevent a diminution in body and mandibular growth and tooth eruption in their offspring.

Acknowledgments. The technical assistant of Ana María Gómez and Nélida Cuello are acknowledged.

\section{REFERENCES}

1. The Expert Committee on the Diagnosis and Classification of Diabetes Mellitus 2002 Report of the Expert Committee on the Diagnosis and Classification of Diabetes Mellitus. Diabetes Care 25(suppl 1):S5-S20

2. Canavan JP, Goldspink DF 1988 Maternal diabetes in rats. Effects on fetal growth and protein turnover. Diabetes 37:1671-1677

3. Eriksson UJ, Dahlstrom E, Hellerstrom C 1983 Diabetes in pregnancy. II. Skeletal malformations in the offspring of diabetic rats after intermittent withdrawal of insulin in early gestation. Diabetes 32:1141-1145

4. Plagemann A, Harder T, Franke K, Kohlhoff R 2002 Long-term impact of neonatal breast-feeding on body weight and glucose tolerance in children of diabetic mothers. Diabetes Care 25:16-22

5. Lau C, Sullivan MK, Hazelwood RL 1993 Effects of diabetes mellitus on lactation in the rat. Proc Soc Exp Biol Med 204:81-89

6. Hartmann P, Cregan M 2001 Lactogenesis and the effects of insulin-dependent diabetes mellitus and prematurity. J Nutr 131:3016S-3020S

7. Bitman J, Hamosh M, Hamosh P, Lutes V, Neville MC, Seacat J, Wood DL 1989 Milk composition and volume during the onset of lactation in a diabetic mother. Am J Clin Nutr 50:1364-1369

8. Neubauer SH, Ferris AM, Chase CG, Fanelli J, Thompson CA, Lammi-Keefe CJ, Clark RM, Jensen RG, Bendel RB, Green KW 1993 Delayed lactogenesis in women with insulin-dependent diabetes mellitus. Am J Clin Nutr 58:54-60

9. Jackson MB, Lammi-Keefe CJ, Jensen RG, Couch SC, Ferris AM 1994 Total lipid and fatty acid composition of milk from women with and without insulin-dependent diabetes mellitus. Am J Clin Nutr 60:353-361

10. Cahill DR, Marks SC Jr 1980 Tooth eruption: evidence for the central role of the dental follicle. J Oral Pathol 9:189-200

11. Wise GE, Marks SC Jr, Cahill DR 1985 Ultrastructural features of the dental follicle associated with formation of the tooth eruption pathway in the dog. J Oral Pathol $14: 15-26$ 
12. Marks SC Jr, Cahill DR, Wise GE 1983 The cytology of the dental follicle and adjacent alveolar bone during tooth eruption in the dog. Am J Anat 168:277-289

13. Ten Cate AR 1998 Hard tissue formation and destruction. In: Oral Histology. Development, Structure and Function. Mosby-Year Book, Inc., St. Louis, pp 69-77

14. Ten Cate AR 1998 Physiological Tooth Movement: Eruption and Shedding. In: Ora Histology. Development, Structure and Function. Mosby-Year Book, Inc., St. Louis, pp 289-314

15. Ubios AM, Braun EM, Cabrini RL 1998 Effect of biphosphonates on abnormal mandibular growth of rats intoxicated with uranium. Health Phys 75:610-613

16. Ubios AM, Piloni MJ, Cabrini RL 1992 Mandibular growth and tooth eruption after localized X-radiation. J Oral Maxillofac Surg 50:153-156

17. De Lucca RC, Mandalunis PM, Ubios AM 1999 Iron overloading inhibits dentine mineralization. Acta Odont Latinoam 12:75-81

18. Pujadas Bigi MM, Lemlich L, Mandalunis P, Ubios AM 2003 Exposure to oral uranyl nitrate delays tooth eruption and development. Health Phys 84:163-169

19. De Lucca RC, Piloni MJ, Villarino ME, Ubios AM 2000 Alterations of tooth eruption in animals treated with EHBP. [abstract] J Dent Res 79:1017

20. Thulesen J, Nexo E, Raaberg L, Poulsen SS 1994 Decreased level of epidermal growth factor in milk from diabetic rats. Pediatr Res 35:107-111

21. Eratalay YK, Simmons DJ, El-Mofty SK, Rosenberg GD, Nelson W, Haus E, Halberg F 1981 Bone growth in the rat mandible following every-day or alternate-day methylprednisolone treatment schedules. Arch Oral Biol 26:769-777

22. Weibel ER, Elias H 1967 Quantitative methods in morphology. In: Weibel ER, Elia $\mathrm{H}$ (eds) Introduction to Stereology and Morphology. Springer Verlag, Berlin, pp 3-19
23. Alippi RM, Meta MD, Olivera MI, Bozzini C, Schneider P, Meta IF, Bozzini CE 2002 Effect of protein-energy malnutrition in early life on the dimensions and bone quality of the adult rat mandible. Arch Oral Biol 47:47-53

24. Partanen AM, Thesleff I 1989 Growth factors and tooth development. Int J Dev Biol 33:165-172

25. Shroff B, Kashner JE, Keyser JD, Hebert C, Norris K 1996 Epidermal growth factor and epidermal growth factor-receptor expression in the mouse dental follicle during tooth eruption. Arch Oral Biol 41:613-617

26. Hoath SB 1986 Treatment of the neonatal rat with epidermal growth factor: differences in time and organ response. Pediatr Res 20:468-472

27. Young WG, Ruch JV, Stevens MR, Bègue-Kirn C, Zhang CZ, Lesot H, Waters MJ 1995 Comparison of the effects of growth hormone, insulin-like growth factor-I and fetal calf serum on mouse molar odontogenesis in vitro. Arch Oral Biol 40:789-799

28. Li H, Bartold PM, Zhang CZ, Clarkson RW, Young WG, Waters MJ 1998 Growth hormone and insulin-like growth factor I induce bone morphogenetic proteins 2 and 4: a mediator role in bone and tooth formation? Endocrinology 139:3855-3862

29. Baumrucker CR, Hadsell DL, Blum JW 1993 Effects of dietary insulin-like growth factor I on growth and insulin-like growth factor receptors in neonatal calf intestine. J Anim Sci 72:428-433

30. Work Group on Breastfeeding, American Academy of Pediatrics 1997 Breastfeeding and the use of human milk. Pediatrics 100:1035-1039

31. Gold AE, Reilly C, Walker JD 1998 Transient improvement in glycemic control. The impact of pregnancy in women with IDDM. Diabetes Care 21:374-378 